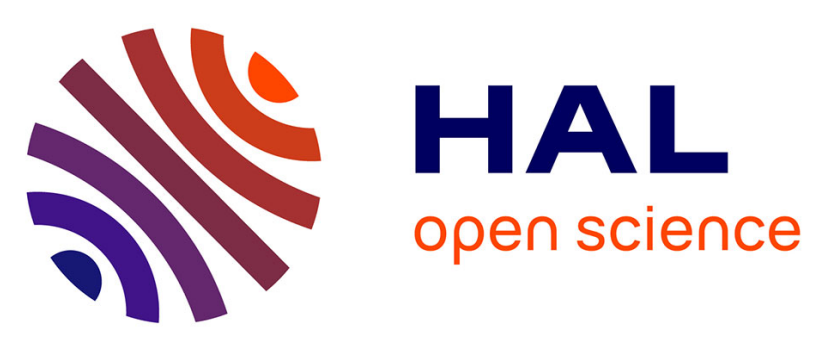

\title{
Representation and self-configuration of physical entities in extended smart grid perimeter
}

Zheng Hu, Gilles Privat, Stéphane Frénot, Bernard Tourancheau

\section{To cite this version:}

Zheng $\mathrm{Hu}$, Gilles Privat, Stéphane Frénot, Bernard Tourancheau. Representation and selfconfiguration of physical entities in extended smart grid perimeter. IEEE PES Innovative Smart Grid Technologies (ISGT) Europe Conference, Oct 2012, Berlin, Germany. hal-00748526

\section{HAL Id: hal-00748526 \\ https://hal.inria.fr/hal-00748526}

Submitted on 5 Nov 2012

HAL is a multi-disciplinary open access archive for the deposit and dissemination of scientific research documents, whether they are published or not. The documents may come from teaching and research institutions in France or abroad, or from public or private research centers.
L'archive ouverte pluridisciplinaire HAL, est destinée au dépôt et à la diffusion de documents scientifiques de niveau recherche, publiés ou non, émanant des établissements d'enseignement et de recherche français ou étrangers, des laboratoires publics ou privés. 


\title{
Representation and Self-Configuration of Physical Entities in Extended Smart Grid Perimeter
}

\author{
Zheng Hu, Gilles Privat, Stéphane Frénot and Bernard Tourancheau
}

\begin{abstract}
Positing the extension of Smart Grids to Smart Energy Management Systems in relevant domains, we propose a framework and a set of mechanisms for the identification, selfconfiguration and representation of a comprehensive range of energy-relevant entities in these target domains. These entities get represented by proxy software components, making up a domain abstraction layer that gets coupled to domain entities through a shared set of networked sensors and actuators. The proposed mechanisms make it possible to configure these entities as if they were state of the art networked devices, provided their relevant models are retrieved from a domain-specific ontology. We describe these mechanisms and their implementation on examples from the home domain.
\end{abstract}

Index Terms-Smart Grid, Microgrid, Energy Management System, Internet of Things, Ontology, Self-configuration.

\section{INTRODUCTION}

$\mathbf{T}$ HE smaller scales of the smart grid may involve the decentralized management of semi-autonomous units such as homes, buildings and district microgrids, where all kinds of electrical equipment and energy-relevant physical entities can get integrated in a local energy management system. These entities may thus be considered to be directly or indirectly connected to a perimeter of the grid that is extended to these district, building and home sub-grids This conceptual extension upholds a proper separation of concerns between the grid at large and the nested sub-grids, wherein a local energy management system serves as a single interface between the nested scales, an interface that serves to hide the individual entities connected in a subgrid from the upper level of hierarchy. A risk of complexity explosion being thus averted, there is no limit to the number of entities that can get connected, directly or indirectly, to a grid that is extended in this way. Such entities from e.g. the home and building domains are much more numerous and heterogeneous than the classical type of power equipment that normally gets directly connected to the distribution network. Besides, their attachment to home, building and district microgrids involves not only an electrical connection, but also their integration in an overlay data network that, together with a local data management and control center, makes up the ICT part of the smart grid at these scales.

This work was supported by Agence Nationale de la Recherche under the ReActivHome project (Habisol Programme).

Z. $\mathrm{Hu}$ is with Orange Labs and INSA-Lyon/CITI (email: zheng.hu@orange.com).

G. Privat is with Orange Labs, 28 Chemin du Vieux Chêne 38240 Meylan, France (email: gilles.privat@ orange.com).

S. Frénot is with INSA-Lyon/CITI and INRIA, INSA de Lyon 6 avenue des Arts, 69621 Villeurbanne CEDEX(email: stephane.frenot@insa-lyon.fr)

B. Tourancheau is with Université Joseph Fourier/LIG, 681 rue de la Passerelle 38400 St Martin dHères (email: bernard.tourancheau@imag.fr).
It is essential to the viability of this extended decentralized smart grid approach that the integration of these entities does not require manual configuration. We investigate in this paper what the self-configuration and representation of these entities in the ICT network coupled to the grid at relevant scales may involve, and propose a novel approach to address it.

\section{RELATED WORK}

A number of projects have, in the past few years, addressed ICT-based energy optimization at scales ranging from the home to district microgrids. Many of these projects restrict the scope of entities that are managed by these systems, or the scope of energy management use cases they address, such as monitoring or demand management. The ReActivHome project addressed exclusively the home environment but has attempted to broaden the scope to all energy-relevant entities ranging from rooms to legacy appliances while addressing the self-configuration of these entities in a "Home Abstraction Layer" [1]. The articulation between smart homes and smart grids has also been addressed by several other projects. K. Kok et al. [2] lay out the possibilities offered by homes that become active peers in smart electricity networks while providing an approach for integrating a smart home into the smart grid by using Web services [3]. D. Niyato et al. [4] show how the smart grid connects with smart home appliances by M2M technologies.

Service-level distributed software infrastructures with zeroconf device/service discovery and representation capabilities such as $\mathrm{UPnP}^{1}$ and DPWS are an obvious inspiration for the solution presented here. Kunshiro et al. [5] propose a residential gateway controller with Plug \& Play mechanism, even if they are limited to equipment endowed with regular network interfaces.

Another research strand has addressed the network integration of low-end physical entities, so as to possibly extend the perimeter of networks beyond regular networked devices such as sensors, by encompassing as network nodes all kinds of physical things that have neither an interface in a digital networking protocol, nor a universal identification in a scheme such as RFID [6]. A similar distinction between entity and "device" has been adopted by the IoT- $\mathrm{A}^{2}$ (Internet of Things Architecture) project [7], and the IoT chapter of the FI-ware ${ }^{3}$ project, according to which an entity may be any physical thing such as an appliance or a room in a home or an HVAC

\footnotetext{
${ }^{1}$ www.upnp.org

${ }^{2}$ www.iot-a.eu

${ }^{3}$ www.fi-ware.eu
} 
system in a building, and devices such as sensors and actuators are intermediaries used for monitoring and controlling these entities which are the proper target for IoT applications such as energy management.

Well ahead of the rollout of smart grids, the power engineering community has standardized the semantic-level Common Information Model [8] (CIM) as a set of models for electrical entities in the same sense, with the aim to support semantically-based self-configuration and interoperability of these entities when they get connected to the smart grid. These models are so far limited to classical EE entities and do not extend, as we propose here, to the home or building domains, Preliminary research work [9][10][11] has also investigated the use of the semantic web for the smart grid.

\section{Proposed FramewORK AND APPROACH}

\section{A. Overall system, target entities and devices}

Extending the domain/zone distinction set out by the Smart Grid Architecture Model [12], our work focuses on microgrid and customer premises domains such as homes and buildings. The problem addressed by our proposed approach can be stated in the most general possible way as shown in Fig. 1: for each targeted domain, an ICT-based energy management or microgrid control system is set up that comprehensively monitors \& controls all energy-relevant physical entities within this domain.

We propose a Domain Abstraction Layer (xAL), where the domain can be a Home (HAL), a Building (BAL) or a District (DAL) as an analogue of Hardware Abstraction Layers used by operating systems: it hides the specifics of the domain hardware beneath a set of generic models and interfaces, acting as a generic informational interface to the physical system.

Absent any native ICT network interface on most of these entities, sensor and actuator devices are available as intermediaries to effect this monitoring \& control of target physical entities, providing an indirect distributed coupling between the ICT system and the target smart grid domain. The most obvious type of such sensor-actuator device is, for the home domain at least, a smart receptacle-plug inserted in the mains connection of the entity, if it is a regular electrical appliance. This device monitors the electrical consumption of the entity as a sensor and may also be used to turn it on or off as an actuator. Yet relevant sensors and actuators are by no means limited to this all too obvious example and any sensor available in the target environment may be used for monitoring entities, at least as a source of contextual information.

As for the home domain, the target entities of the home are defined as the components of the home that are relevant for being controlled and monitored by an application such as energy management, but also for home security or home automation, using the ICT system that is being setup in this way as a generic supporting layer.

The ICT system will "shadow" these physical entities individually through matching self-contained ICT subsystems that will in turn be the primary building blocks of its own architecture. The ICT system should have the capability to create and configure these entities automatically, both for the

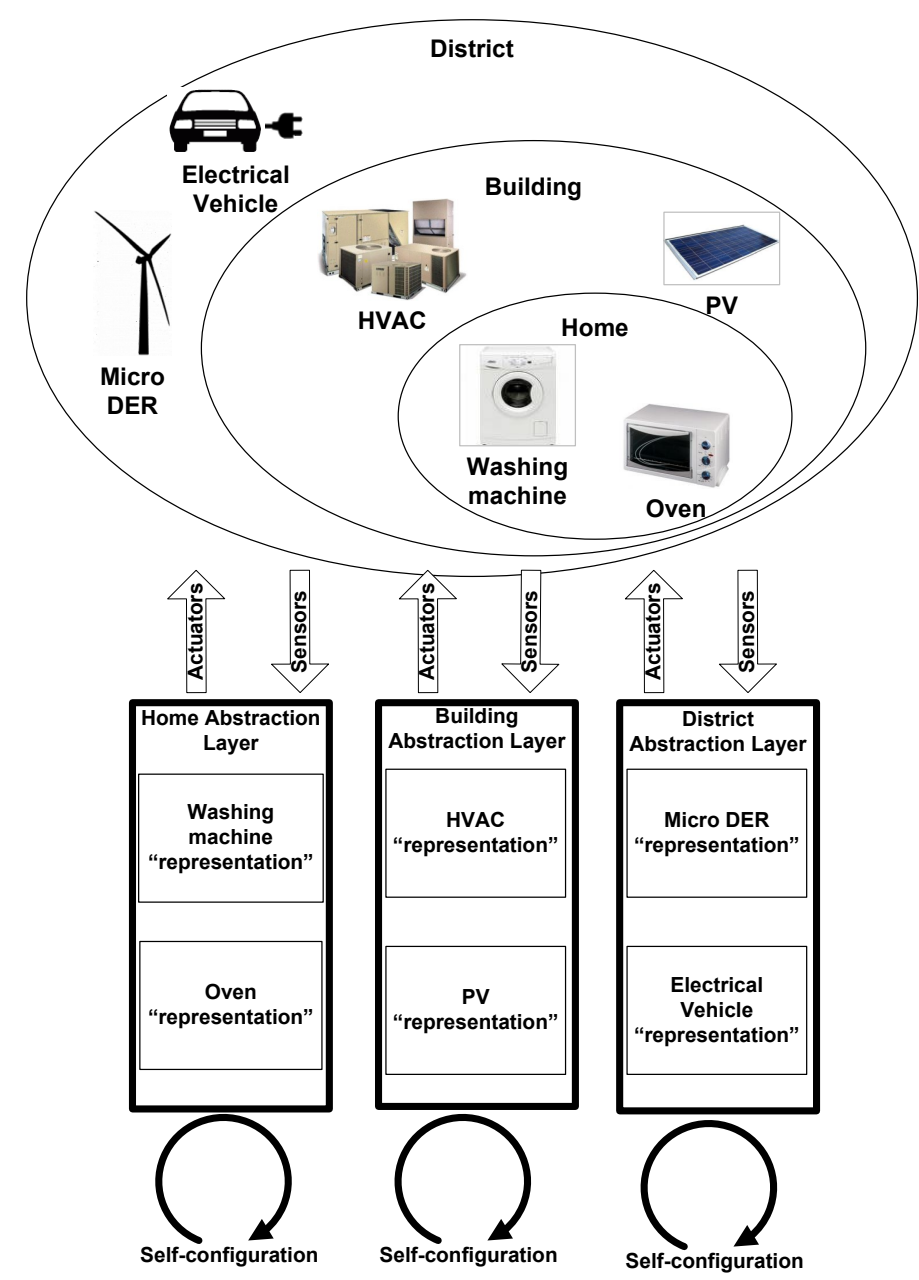

Fig. 1. Principle of the proposed approach in nested grid domains

initial configuration stage and when reconfiguration is needed because of a change in the environment. This implies the need to automatically associate the subset of sensors and actuators that are used as intermediaries for the monitoring and control of a given entity, and to update the model of the subsystem accordingly.

The proposed approach is to dynamically create a proxy or representation ICT component for each individual target physical entity of the home. We call this component the Entity Identification, Monitoring \& Control component (EIMC) in the following. It connects to the physical subsystem through a dynamically configured set of potentially shared sensors and actuators, chosen among those available in the relevant environment.

The sensors detect events and changes of state from the physical entity as their inputs and the EIMC sends the controls to the actuators to effect required actions on the physical entity. The system comprising the EIMC together with the physical entity and the sensors and actuators makes up a closed-loop 
control system.

\section{B. Domain entity ontology}

Our approach assumes the prior definition of an ontology or model repository, similar to CIM [8], that captures generic knowledge about the entities of the target domain through a multi-criteria hierarchical categorization and the definition of minimal generic finite state models for each of the target entities. Initiating and configuring the EIMC for a given entity actually amounts to identifying, loading and iteratively adapting the most appropriate entity model from this model repository by traversing the graph that represents parentdescendant relationships between models.

This ontology subsumes multiple relevant categorizations of the target entities, in the context of the target domain. As illustrated in Fig. 2 for the example of a home-domain ontology of appliances it can be structured as a directed acyclic graph that makes it possible to follow a traversal path from the most generic parent models (closer to the roots of the graph) to the more specific models (closer to the leaves). At the root is the main class Entity, as understood above, which in the scope of the building domain, specializes further into two descendant categories : energy-relevant pieces of equipment (appliances, etc) and the subsets of space which contain this equipment. Appliances can in turn be specialized and classified according to several different criteria that are either intrinsic to their main usage, or relative to the application (in our case energy management and energy efficiency). Examples of these criteria are illustrated below, each of them corresponding to intermediate nodes in the graph.

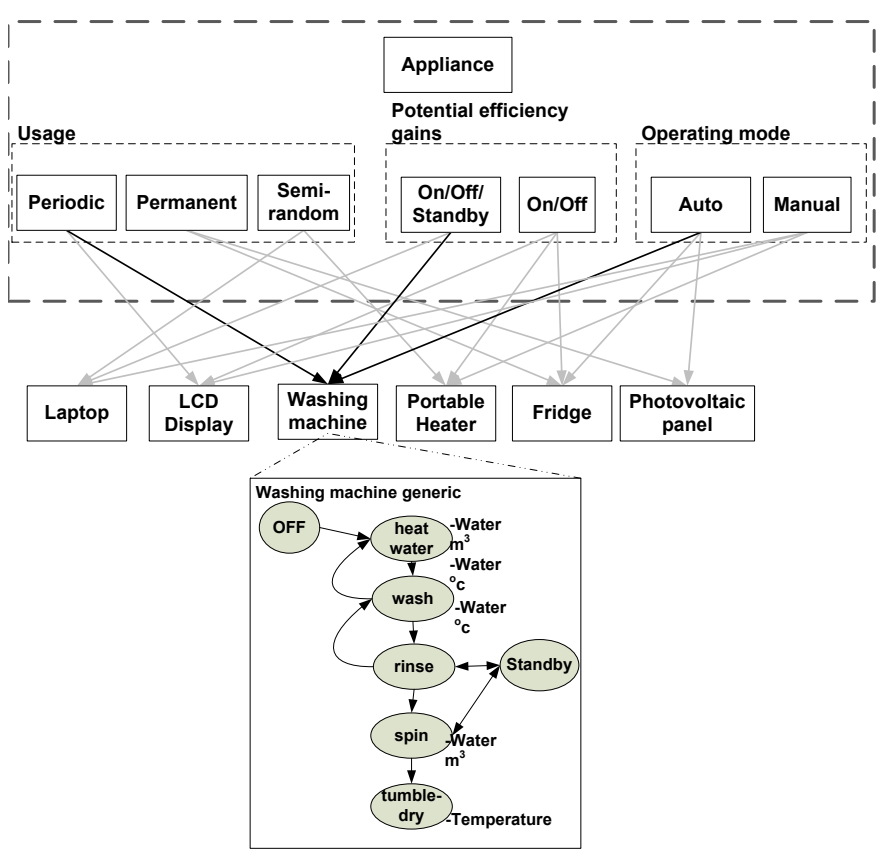

Fig. 2. Home entity ontology with energy-relevant categorizations and corresponding discrete state model example for home appliances

Models are associated not only with the leaf nodes of the graph corresponding to the most specific categories, but also to intermediate nodes. This full hierarchy of models provides a mechanism to identify an entity in an incremental way on the basis of observation data, starting from the most generic model if observed sensor data is inconclusive, and refining this first match to more specific models down the graph when further observation data become available.

We chose to model target entities of the home domain with hybrid finite-state discrete-time models, where state information is possibly complemented with continuous-valued attributes. These states and the relevant attributes are then stored as the state of the shadow ICT EIMC. These hybrid models represent a tradeoff between expressivity and ease of identification, the full description of physical systems such as the target appliances would normally require a continuous state \& continuous-time model, but the automatic identification of the parameters of such models would be impossible. An example of such a simple model is given in Fig. 2 for a washing machine.

As construed above, subsets of the building space can also be considered as relevant target entities for inclusion in the energy management system perimeter. In the home domain, a single room can be modeled, in a way similar to what is done with appliances, as shown in Fig. 3. At larger spatial scales, in a city-district domain, an energy-relevant equipment entity could be a micro_DER, and a corresponding space entity could be a street or block of buildings.

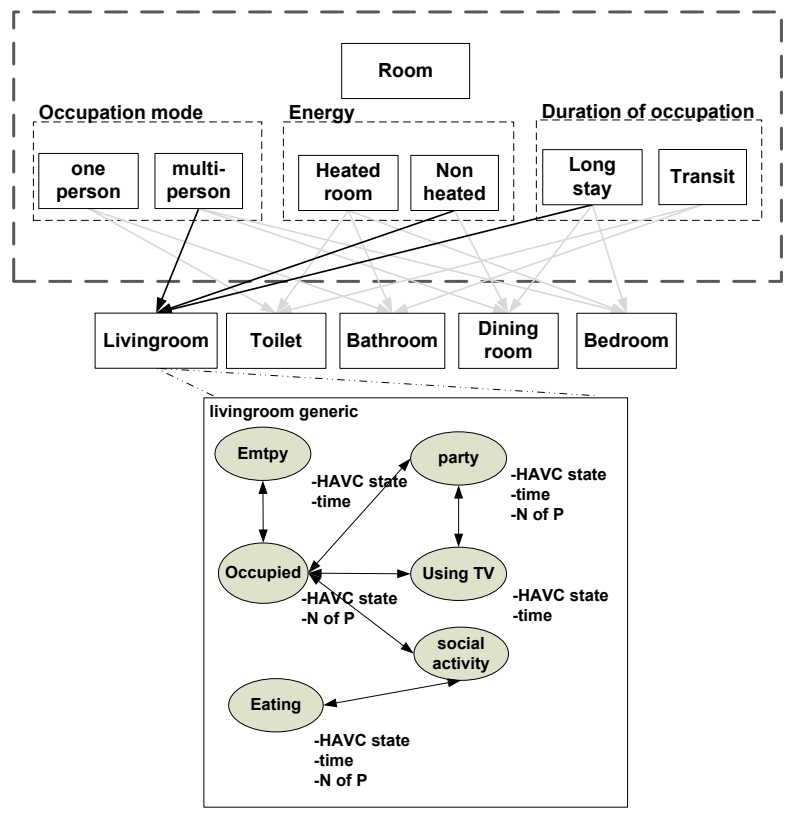

Fig. 3. Home entity ontology and corresponding discrete state model example for rooms

\section{Example scenario}

We assume a home end-user connects a new washingmachine to the grid through a smart receptacle-plug, as mentioned above. From this sensor association, the washingmachine can first be identified as a mains-connected electrical appliance, and integrated with a minimal electrical state model 
(ON/OFF) in the HAL, making it available to an energy management application to be controlled or monitored. As more data is gathered from the use of this appliance, the system can identify it as a washing machine with a more accurate model as shown in Fig. 2. By using this model, the system can provide richer energy consumption information associated with the different states, and make it possible to control the appliance according to the state it is in at any given time.

Once all physical entities are configured and represented in the local energy management system in this way, their energyrelevant information can be updated in real-time. Compared to smart metering [13], a much richer and finer information is available, as it is not only the aggregate energy consumption information that can be collected, but also the detailed energyrelated behavior of each individual energy-relevant physical entity that can be separately maintained in the domain energy management system, making it possible for this system to act as an intermediary between the local domain and the grid at large to control the individual physical entities through their representation proxy (EIMC) for, e.g. implementing demand management by responding to grid-originating signals, or performing local optimization and source-load balancing.

\section{Abstraction Layer Configuration}

After describing the overall framework of our proposed approach, we present in this section the overall architecture and detail the configuration mechanisms of the Abstraction Layer and individual EIMCs.

Elaborating on the solution described above in its principle, we present its more detailed mapping onto an actual information system. The overall information system comprises, as shown in Fig. 4, an application layer that is supported by the more generic abstraction layer, which comprises a set of EIMC instances coupled to the physical entity they control and monitor. This layer is the object of the present invention. The application layer contains the application which can use the data and functionalities provided by the abstraction layer to monitor and control the target entity. The abstraction layer acquires stores and generates information useful for application layer through each EIMC.

The architecture shown in Fig. 4 is for one EIMC instance within the EIMC layer. The key role of the EIMC, as mentioned above, is to represent an abstraction of a physical entity such as a home appliance in the information system. We can deal directly with the EIMC to retain an up-to-date knowledge of the state that the physical entity is in; we can also transmit control information to actuators associated to the EIMC to effect actions on the target physical entity. And as the EIMC system is a closed-loop system, it can also act as an autonomous control system to regulate locally the physical entity without intervention from the external application layer.

\section{A. Model identification and parametrization}

Model identification is the process of matching a target entity to a category (a node of the graph) in the domainspecific entity ontology. This category may be very broad or

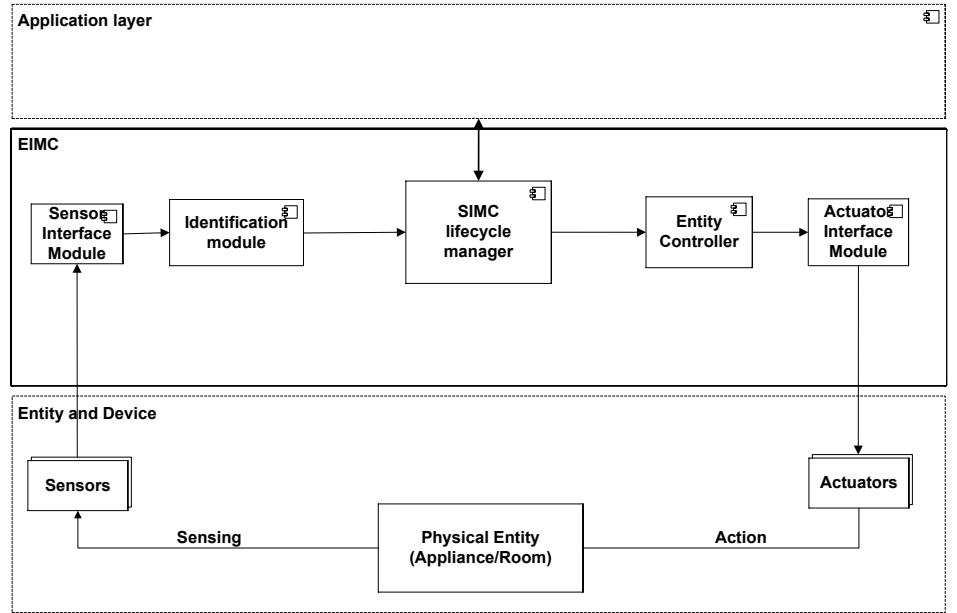

Fig. 4. EIMC functional architecture

very specific, depending on how much sensor data the system has available to perform the identification with. This category is associated with a default model of the target entity.

Once a given model has been identified, parameters for the corresponding model may be updated incrementally from new sensor data, or the model may be modified so as to better fit observations: this may correspond to either changing continuous-valued state attributes, invalidating state transitions, or removing states from the state diagram.

An example of this updating process is shown in Fig. 5 for a washing machine, where the final (most specific) model reached in the model identification stage is refined by removing an unused state or an unused transition (in this case the washing machine is updated from the generic one as having no tumble-drying function) Adding new states is not considered possible here, and if the model does not fit the observations it is always possible to revert to the previous stage, starting with a new model one rung higher (i.e. less specific) in the hierarchy.

\section{B. EIMC configuration and runtime}

We now detail the EIMC life-cycle and configuration mechanism involved in EIMC creation. As mentioned above, Fig. 5 presents the finite-state machine model used by a washing machine EIMC. The EIMC can be in either of two main modes: configuration or runtime. The configuration phase is used to associate the kind of specific state automata that best represents the appliance, in our concept it is called Runtime State Machine (RSM). During the runtime phase, it represents not only the states where the EIMC may be in but also but also checks the conformance via the state sequence matching. The configuration phase consists in associating sensors, actuators and a Runtime State Machine (RSM). There are three predefined states. The newborn state is active when the existence of a physical washing machine has just been discovered from a set of sensors. The configuring state is active when a dedicated washing machine EIMC instance is associated with real sensors/actuators and its RSM. The destroying state is active when the physical entity is removed 


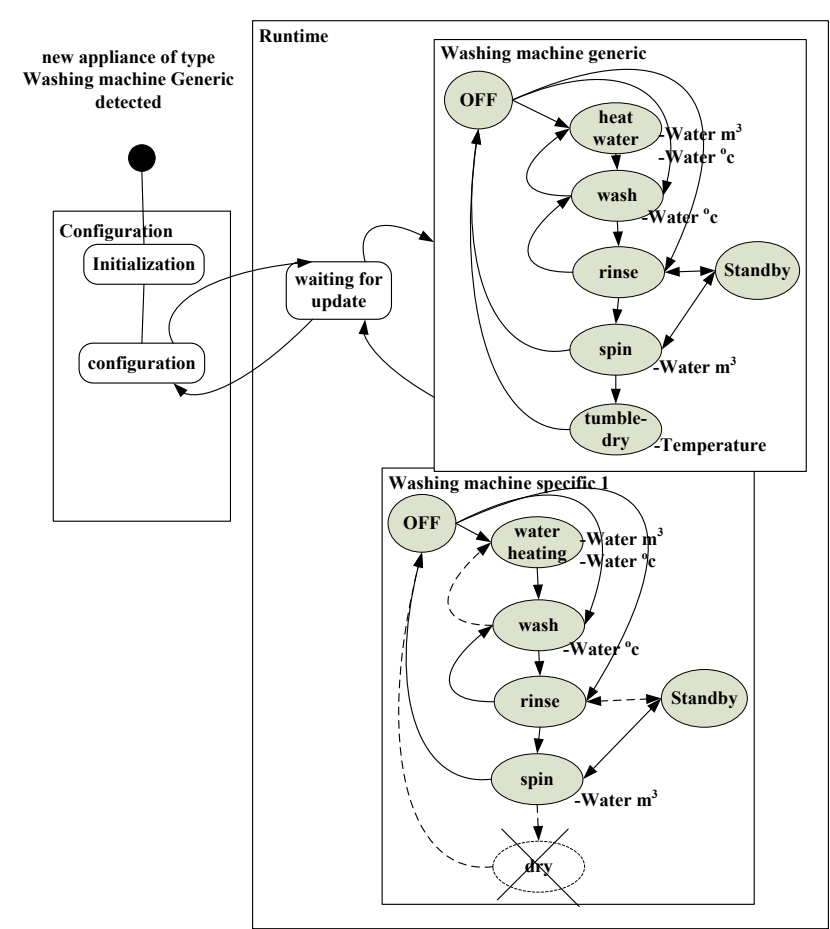

Fig. 5. Model update example

from the home or disconnected from the system; it corresponds to the deletion of the corresponding EIMC instance from the system.

The entity Generic Runtime maintains as persistent variables the current states of the EIMC while the physical entity works. For example ON/OFF states are specific to the generic kind of detected appliance. In runtime mode the EIMC component may always go to the waiting for synch state when an error occurs, or when the state is unknown.

A reconfiguration stage may occur at anytime. For instance if we found there is no dry state for the entity, we go back in configuring mode, change values and set the EIMC in the waiting for synch state.

\section{EIMC identification engine}

EIMC identification engine is a fundamental element of the system. As a identification or pattern recognition engine, it selects the best approximation from a multidimensional dataset of sensor readings. Various algorithms from the machine learning repertoire may be used for this. Based on this principle, it is used in two cases: the identification of the best approximating model of the target physical entity and the identification of the instantaneous state of the target entity according to this model. For instance, at the beginning, the recognition engine should identify the type of appliance detected and spawn a generic washing machine model. Then the new sensor data is used to find the best match which state the washing machine is in at any given time, matching it to one of the defined states of the automaton.

\section{IMPLEMENTATION}

\section{A. Smart Home abstraction layer implementation}

We describe a complete implementation architecture of our proposed approach, taking the home domain as an example.

The Home Abstraction Layer comprising the EIMC management system is hosted on a dedicated home server called Home Automation Box (HAB), itself connected to a wireless network of sensors and actuators (WSAN) inside the home. As shown in Fig. 6, at the bottom of the HAB architecture, a sensor/actuator interface layer provides an abstraction of the various types of connected sensors and actuators [14]. The upper layer of the HAL maintains a virtual representation of each physical entity as a distinct EIMC instance. Those representations interface home applications, among which smart grid-oriented energy management, with the managed physical entities.

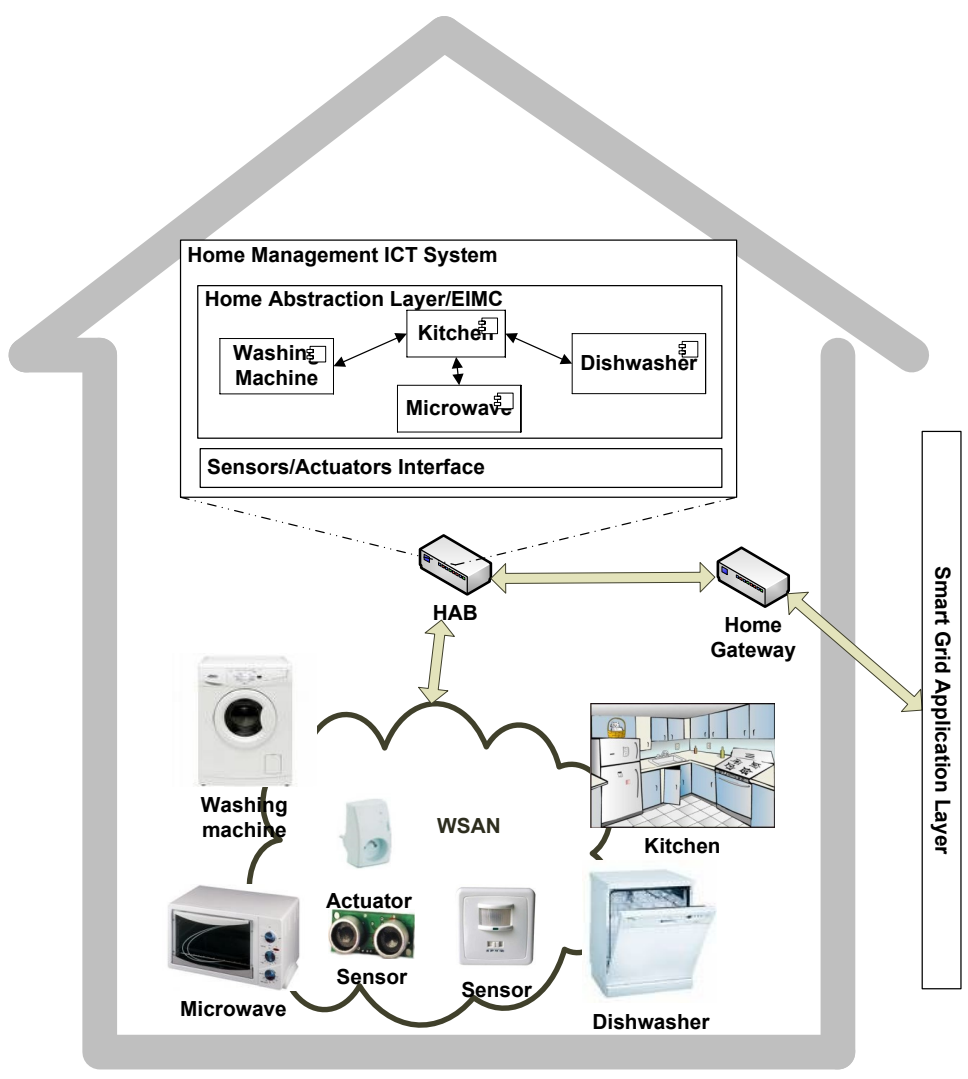

Fig. 6. The home abstraction layer management and communication architecture

Through a single point of interface going through this energy management system, the home is thus integrated into nesting energy systems (e.g. building, microgrid) and the power grid at large.

As mentioned above, each EIMC is associated with a set of sensors and actuators, which are shared between different entities. In the home context, EIMCs associated with rooms are linked with those associated with appliances in this room in the sense that a room EIMC can use a heating EIMC as one of its associated actuators to effect changes in temperature, or use another appliance EIMC as a presence sensor. Our architecture 
assumes that the sensor-actuator network is available and has been deployed beforehand. Contrary to these "generic" sensors and actuators, the EIMC of an appliance used as a sensor or actuator is configured during the initialization phase of the system.

\section{B. OSGi-based implementation}

The OSGi specification [15] defines a standardized, component-oriented computing environment for embedded networked services that can serve as the foundation for a service oriented architecture. Among the benefits of choosing OSGi is to the possibility to support application reconfiguration at runtime. Each EIMC functional module is modeled as a bundle, and its functionalities are packaged in the bundle as services.

To achieve the full functionality described in section IV, we model the architecture of the EIMC management system as shown in Fig. 7. Three bundles are initially started:

The Sensor interface Module bundle communicates with all sensors in the HAL environment. Each piece of sensor data is transferred by the sensor interface module which achieves the following tasks:

1) Physical interface for heterogeneous sensor management [14];

2) Identification and authentication of data source;

3) Cartography of the observed environment;

4) Dynamic integration of sensors according to their own protocol, for which we mostly (not exclusively) use Zigbee [16].

The Actuator interface Module, counterpart of the Sensor interface Module, takes in high-level commands expressed as state changes of target physical entities and fans them out as specific controls to the associated actuators.

The Global Context Monitor bundle waits for the activation of the Sensor interface Module and Actuator interface Module, in order to start creating the EIMC. The physical system configuration information is communicated by this module to the application in charge of managing the corresponding entity.

EIMC comprises the following operating services:

1) The Life-cycle service is the core of EIMC, it is capable of starting or stopping EIMCs. It is invoked by the Global Context Monitor, and requests the creation of a defined type of entity corresponding to a set of sensed data;

2) Identification is based on a model which produces a unique output from various sensor inputs of sensed data in a single classifier. This output can be transferred to RSM;

3) 3) RSM service maintains the current state by receiving notification from the identification engine, and also for state sequence conformance checking. It can communicate with the upper layer to provide the physical entity information as well as receive commands for the physical entity;

4) The OntologyManager service can load the pre-defined physical entity model from a external online generic model repository such as CIM [8].

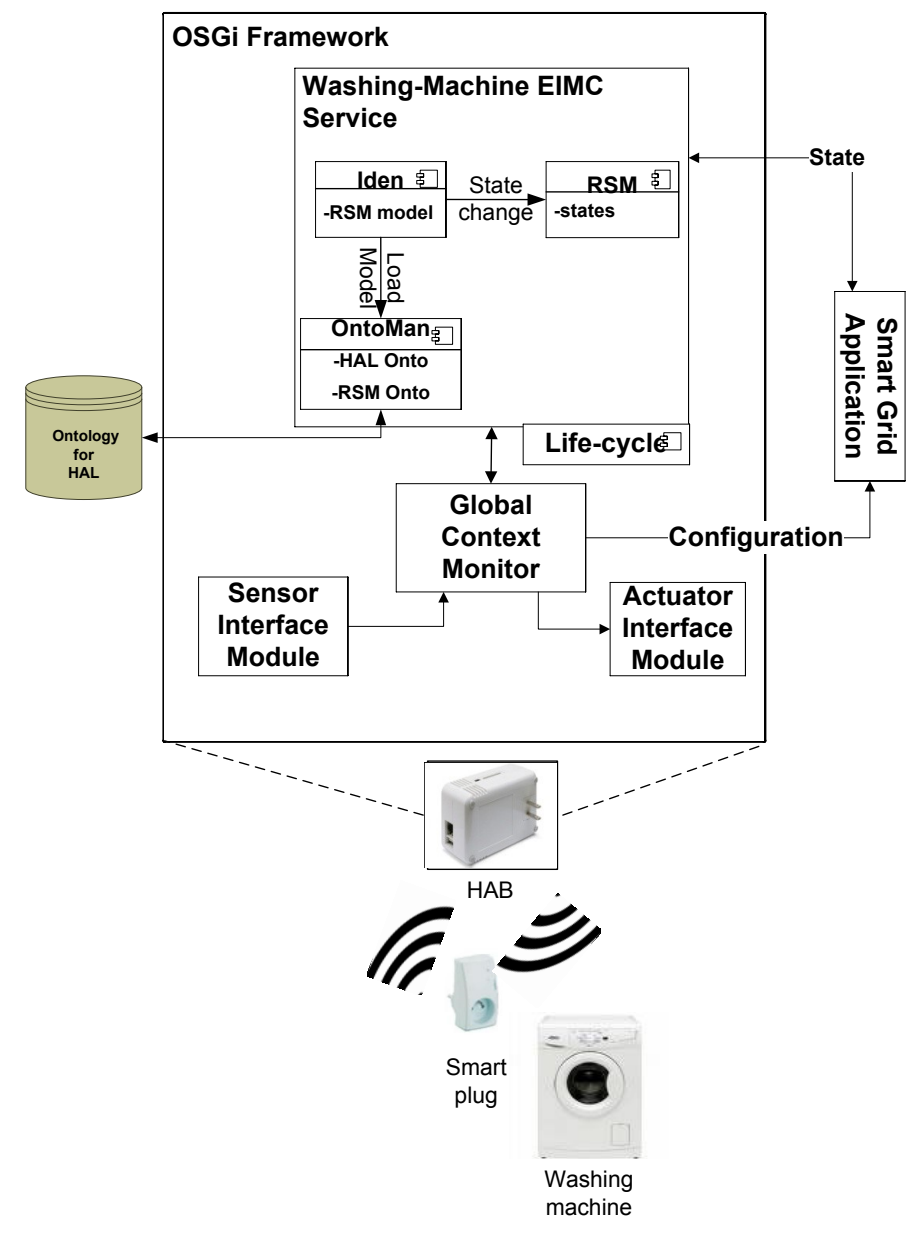

Fig. 7. EIMC OSGi-based implementation example

\section{CONCLUSION AND FUTURE WORK}

The proposed approach has been developed and validated so far in the home domain, on the basis of a complete range of home entities, from electrical appliances to rooms. If rightly viewed as an extension of the Internet of Things [6], nothing in this approach is inherently limited to these entities and this domain, and the next steps of our work will extend this validation to the non-residential building and city-district domains. Encompassing such a diverse range of entities in a comprehensive energy management system at these scales may appear to go well beyond the received notion of what smart grid is, yet it is an all to natural extension of the original idea of coupling ICT and electricity towards coupling ICT-and the physical environment, inasmuch as the physical entities it comprises are relevant to the same purpose of smart energy management, a management that proper separation of concerns delegates to this local scale. 


\section{REFERENCES}

[1] Z. Hu, G. Privat, S. Frenot, and B. Tourancheau, "Self-configuration of home abstraction layer via sensor-actuator network," in Ambient Intelligence, ser. Lecture Notes in Computer Science. Springer Berlin Heidelberg, 2011, vol. 7040, pp. 146-150.

[2] K. Kok, S. Karnouskos, D. Nestle, A. Dimeas, A. Weidlich, C. Warmer, P. Strauss, B. Buchholz, S. Drenkard, N. Hatziargyriou, and V. Lioliou, "Smart houses for a smart grid," in Electricity Distribution - Part 1, 2009. CIRED 2009. 20th International Conference and Exhibition on, june 2009, pp. $1-4$.

[3] C. Warmer, K. Kok, S. Karnouskos, A. Weidlich, D. Nestile, P. Selzam, J. Ringelstein, A. Dimeas, and S. Drenkard, "Web services for integration of smart houses in the smart grid," in Grid-Interop The road to an interop erable grid, november 2009.

[4] D. Niyato, L. Xiao, and P. Wang, "Machine-to-machine communications for home energy management system in smart grid," Communications Magazine, IEEE, vol. 49, no. 4, pp. 53 -59, april 2011.

[5] N. Kushiro, S. Suzuki, M. Nakata, H. Takahara, and M. Inoue, "Integrated residential gateway controller for home energy management system," Consumer Electronics, IEEE Transactions on, vol. 49, no. 3, pp. $629-636$, aug. 2003.

[6] G. Privat, "Phenotropic and stigmergic webs: the new reach of networks," Universal Access in the Information Society, pp. 323-335, 2012.

[7] S. De, P. Barnaghi, M. Bauer, and S. Meissner, "Service modelling for the internet of things," in Computer Science and Information Systems (FedCSIS), 2011 Federated Conference on, sept. 2011, pp. 949 -955.

[8] A.W.Mcmorran, "An introduction to iec 61970-301 \& 6196811 the common information model," 2008. [Online]. Available: https://cimphony.com/cim-intro.pdf

[9] R. King, "Information services for smart grids," in Power and Energy Society General Meeting - Conversion and Delivery of Electrical Energy in the 21st Century, 2008 IEEE, july 2008, pp. $1-5$.

[10] S. Rohjans, M. Uslar, and H. Juergen Appelrath, "Opc ua and cim: Semantics for the smart grid," in Transmission and Distribution Conference and Exposition, 2010 IEEE PES, april 2010, pp. 1 -8.

[11] I. Lendak, E. Varga, A. Erdeljan, and M. Gavric, "Restful web services and the common information model (cim)," in Energy Conference and Exhibition (EnergyCon), 2010 IEEE International, dec. 2010, pp. 716 $-721$.

[12] "Report on the reference architecture for the smart grid. draft version," 2012.

[13] S. S. S. R. Depuru, L. Wang, and V. Devabhaktuni, "Smart meters for power grid: Challenges, issues, advantages and status," Renewable and Sustainable Energy Reviews, vol. 15, no. 6, pp. 2736 - 2742, 2011.

[14] L. Gurgen, C. Roncancio, C. Labbé, A. Bottaro, and V. Olive, "Sstreamware: a service oriented middleware for heterogeneous sensor data management," in Proceedings of the 5th international conference on Pervasive services, ser. ICPS '08. New York, NY, USA: ACM, 2008, pp. 121-130.

[15] OSGi, "Osgi service plateform core specification release 4 version 4.2," 2009. [Online]. Available: http://www.osgi.org

[16] Y.-G. Ha, "Dynamic integration of zigbee home networks into home gateways using osgi service registry," Consumer Electronics, IEEE Transactions on, vol. 55, no. 2, pp. $470-476$, may 2009.

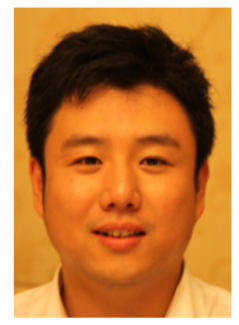

Zheng Hu received Engineering degree from INSALyon, Department of Electrical Engineering in 2009 and is currently working towards his $\mathrm{PhD}$ degree in Orange-labs France Telecom R\&D and CITI laboratory INSA-Lyon. His research interests include distributed system architecture, Self-configuration for IOT, microgrids.

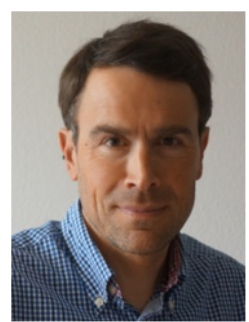

Gilles Privat received engineering and doctoral degrees in systems theory from Télécom ParisTech Institute. He has been a research associate with CNRS and a research engineer and head of a research group with CNET, a public research laboratory in telecommunications. He is currently a senior scientist and project leader with Orange Labs (formerly France Telecom R\&D), where he pioneered the research domain of "smart devices" that has henceforth branched out into Ambient Intelligence/Smart Spaces and M2M/Internet of Things. He is still pursuing a cross-domain range of expertise in this purview, with a current focus on applications of the IoT to home energy management and smart energy. He has authored or co-authored more than 60 peer-reviewed publications and holds 12 patents.

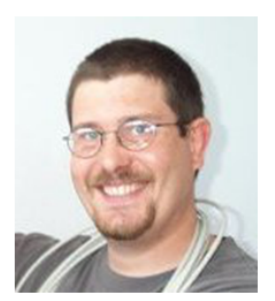

Stéphane Frénot is full professor at the Center for Innovation in Telecommunication and Integration of Services (CITI Lab.) of Lyon University (INSA Lyon, France). His main interests are middlewares, component instrumentation, component security, deployment and management in constrained devices and especially home gateways. His main research domain is targeted around OSGi architecture extensions and virtual machines.

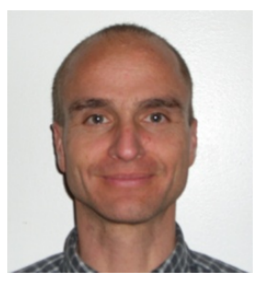

Bernard Tourancheau got MSc in Apply Maths from Grenoble University and an MSc in Renewable Energy Science and Technology from Loughborough University. He was awarded best Computer Science $\mathrm{PhD}$ by Institut National Polytechnique of Grenoble in 1989 for his work on Parallel Computing for Distributed Memory Architectures. He was appointed Professor at University Joseph Fourier of Grenoble in 2012. Since then, he is developing research about protocols and architectures for sensor networks and their applications to building energy efficiency in the LIG laboratory Drakkar team. He has authored more than an hundred peerreviewed publications and owns 8 patents. 